\title{
Attractors for the nonclassical reaction-diffusion equations on time-dependent spaces
}

\author{
Kaixuan Zhu ${ }^{1 *}$ (D), Yongqin Xie ${ }^{2}$ and Feng Zhou ${ }^{3}$
}

\section{"Correspondence:}

zhukx12@163.com

${ }^{1}$ Hunan Province Cooperative

Innovation Center for the

Construction and Development of

Dongting Lake Ecological Economic

Zone, College of Mathematics and

Physics Science, Hunan University of

Arts and Science, Changde, P.R.

China

Full list of author information is available at the end of the article

\begin{abstract}
In this paper, based on the notation of time-dependent attractors introduced by Conti, Pata and Temam in (J. Differ. Equ. 255:1254-1277, 2013), we prove the existence of time-dependent global attractors in $\mathcal{H}_{t}$ for a class of nonclassical reaction-diffusion equations with the forcing term $g(x) \in H^{-1}(\Omega)$ and the nonlinearity $f$ satisfying the polynomial growth of arbitrary $p-1(p \geq 2)$ order, which generalizes the results obtained in (Appl. Anal. 94:1439-1449, 2015) and (Bound. Value Probl. 2016: 10, 2016).
\end{abstract}

MSC: 35R10; 35B40; 35B41

Keywords: Nonclassical reaction-diffusion equations; Polynomial growth of arbitrary order; Time-dependent global attractors

\section{Introduction}

Let $\Omega$ be a bounded domain in $\mathbb{R}^{n}(n \geq 3)$ with smooth boundary, we consider the longtime behavior of the solutions for the following nonclassical reaction-diffusion equation:

$$
\begin{cases}u_{t}-\varepsilon(t) \Delta u_{t}-\Delta u+f(u)=g(x) & \text { in } \Omega \times(\tau, \infty), \\ u=0 & \text { on } \partial \Omega \times(\tau, \infty), \\ u(x, \tau)=u_{\tau}, & x \in \Omega,\end{cases}
$$

where $t>\tau, \tau \in \mathbb{R}$ is the initial time, $g(x) \in H^{-1}(\Omega)$ is an external force term, $\varepsilon(t) \in C^{1}(\mathbb{R})$ is a decreasing bounded function satisfying

$$
\lim _{t \rightarrow+\infty} \varepsilon(t)=0
$$

and there exists $L>0$ such that

$$
\sup _{t \in \mathbb{R}}\left(|\varepsilon(t)|+\left|\varepsilon^{\prime}(t)\right|\right) \leq L .
$$

(c) The Author(s) 2020. This article is licensed under a Creative Commons Attribution 4.0 International License, which permits use sharing, adaptation, distribution and reproduction in any medium or format, as long as you give appropriate credit to the original author(s) and the source, provide a link to the Creative Commons licence, and indicate if changes were made. The images or other third party material in this article are included in the article's Creative Commons licence, unless indicated otherwise in a credit line to the material. If material is not included in the article's Creative Commons licence and your intended use is not permitted by statutory regulation or exceeds the permitted use, you will need to obtain permission directly from the copyright holder. To view a copy of this licence, visit http://creativecommons.org/licenses/by/4.0/. 
For the nonlinear term $f \in C(\mathbb{R}, \mathbb{R})$, similar to that in [3,20,24], we make the following classical assumptions:

$$
f^{\prime}(u) \geq-l, \quad \forall u \in \mathbb{R}
$$

and

$$
-c_{0}+c_{1}|u|^{p} \leq f(u) u \leq c_{0}+c_{2}|u|^{p}, \quad p \geq 2
$$

for some positive constants $c_{0}, c_{1}, c_{2}$.

Let $\mathcal{F}(u)=\int_{0}^{u} f(r) d r$, then there are constants $\tilde{c}_{i}>0(i=0,1,2)$ such that

$$
-\tilde{c}_{0}+\tilde{c}_{1}|u|^{p} \leq \mathcal{F}(u) \leq \tilde{c}_{0}+\tilde{c}_{2}|u|^{p}, \quad \forall u \in \mathbb{R} .
$$

For Eq. (1.1), when $\varepsilon(t)>0$ is a constant, the existence and long-time behavior of solutions have been extensively studied by several authors; see, e.g., $[1,4,5,23,25-$ $27,29,30,32]$. In $[4,5,29]$, the authors main considered the existence of solutions for this type of equations. In $[1,23,25-27,30]$, the authors main considered the existence of the global attractors (see [23, 25-27]) and the pullback (or the uniform) attractors (see $[1,23,30]$ ) in $H_{0}^{1}(\Omega)$ (or $H^{1}\left(\mathbb{R}^{N}\right)$ ). In particular, in [32], we obtained the existence of the pullback attractors in $C_{H_{0}^{1}(\Omega)}$ (rather than in $H_{0}^{1}(\Omega)$ ) for the nonclassical reactiondiffusion equations with delays.

When $\varepsilon(t)=0$, Eq. (1.1) becomes the classical reaction-diffusion equation. The existence and the long-time behavior of solutions have also been extensively investigated by several authors; see, e.g., [2, 11, 12, 17, 21, 28, 31]. In [2, 11, 12, 28], the authors mainly considered the existence (or the blowup), uniqueness and the long-time decay of the solutions for the semilinear parabolic equation [11,12], the nonlinear parabolic equation [2] and the coupled parabolic systems [28]. In [17, 21,31], the authors have proved the existence of the global attractors in $L^{p}(\Omega), H_{0}^{1}(\Omega), L^{2 p-2}(\Omega), H^{2}(\Omega)$ (see [31]) and the existence of the pullback attractors in $L^{p}(\Omega)$ and $H_{0}^{1}(\Omega)$ (see [17] and [21], respectively).

When $\varepsilon(t) \in C^{1}(\mathbb{R})$ satisfies (1.2)-(1.3), the long-time behavior of solutions for Eq. (1.1) has been considered by some researchers; see, e.g., [16, 18]. In [16], the authors have proved the existence of the time-dependent global attractors in $\mathcal{H}_{t}$ with the nonlinearity $f$ satisfying $\left|f^{\prime \prime}(u)\right| \leq c(1+|u|)$ (see Theorem 3.4 in [16] for details). Furthermore, in [18], the authors have considered the case of the nonlinearity $f$ satisfying the critical exponent growth and proved the existence of the time-dependent global attractors in $\mathcal{H}_{t}$ (see Theorem 3.3 in [18] for details).

In this paper, we consider Eq. (1.1) with the nonlinearity $f$ satisfying polynomial growth of arbitrary $p-1(p \geq 2)$ order, which makes that the Sobolev compact embedding is no longer valid and brings more difficulty for verifying the corresponding asymptotic compactness of the solutions process $\{U(t, \tau)\}_{t \geq \tau}$. In order to overcome the difficulty mentioned above, we verify the existence of the time-dependent global attractors $\hat{\mathcal{A}}$ in $\mathcal{H}_{t}$ for the process $\{U(t, \tau)\}_{t \geq \tau}$ by applying the contractive function methods as in $[6,13,14,19,22,27]$ (see Theorem 3.8). 


\section{Preliminaries}

In this section, we firstly review briefly some notations, basic definitions and results about processes on time-dependent spaces (see [7-9, 19] for details).

\subsection{Notations}

Let $\left\{X_{t}\right\}_{t \in \mathbb{R}}$ be a family of normed spaces, we introduce the $R$-ball of $X_{t}$ as

$$
\mathbb{B}_{t}(R)=\left\{z \in X_{t}:\|z\|_{X_{t}} \leq R\right\} .
$$

For any given $\epsilon>0$, the $\epsilon$-neighborhood of a set $B \subset X_{t}$ is defined as

$$
\mathcal{O}_{t}^{\epsilon}(B)=\bigcup_{x \in B}\left\{y \in X_{t}:\|x-y\|_{X_{t}}<\epsilon\right\}=\bigcup_{x \in B}\left\{x+\mathbb{B}_{t}(\epsilon)\right\} .
$$

We denote the Hausdorff semidistance of two (nonempty) sets $B, C \subset X_{t}$ by

$$
\delta_{t}(B, C)=\sup _{x \in B} \inf _{y \in C}\|x-y\|_{X_{t}} .
$$

Moreover, we introduce the time-dependent space $\mathcal{H}_{t}$ endowed with the norms

$$
\|u\|_{\mathcal{H}_{t}}^{2}=\|u\|_{2}^{2}+\varepsilon(t)\|\nabla u\|_{2}^{2}
$$

where $\|\cdot\|_{2}$ denotes the usual norm in $L^{2}(\Omega)$.

\subsection{Some concepts}

In this subsection, we give some concepts about the time-dependent global attractors.

Definition 2.1 Let $\left\{X_{t}\right\}_{t \in \mathbb{R}}$ be a family of normed spaces. A process is a two-parameter family of mappings $U(t, \tau): X_{\tau} \rightarrow X_{t}, t \geq \tau, \tau \in \mathbb{R}$ with properties

(i) $U(\tau, \tau)=$ Id is the identity operator on $X_{\tau}, \tau \in \mathbb{R}$;

(ii) $U(t, s) U(s, \tau)=U(t, \tau), \forall t \geq s \geq \tau, \tau \in \mathbb{R}$

Definition 2.2 A family $\hat{C}=\left\{C_{t}\right\}_{t \in \mathbb{R}}$ of bounded sets $C_{t} \subset X_{t}$ is called uniformly bounded if there exists a constant $R>0$ such that $C_{t} \subset \mathbb{B}_{t}(R)$ for all $t \in \mathbb{R}$.

Definition 2.3 A family $\hat{B}=\left\{B_{t}\right\}_{t \in \mathbb{R}}$ is called pullback absorbing if it is uniformly bounded and for every $R>0$, there exists a constant $t_{0}=t_{0}(t, R) \leq t$ such that $U(t, \tau) \mathbb{B}_{\tau}(R) \subset B_{t}$ for all $\tau \leq t_{0}$.

The process $\{U(t, \tau)\}_{t \geq \tau}$ is called dissipative whenever it admits a pullback absorbing family.

Definition 2.4 A time-dependent absorbing set for the process $\{U(t, \tau)\}_{t \geq \tau}$ is a uniformly bounded family $\hat{B}=\left\{B_{t}\right\}_{t \in \mathbb{R}}$ with the following property: for every $R \geq 0$ there exists a $t_{0}=t_{0}(R) \geq 0$ such that

$$
U(t, \tau) \mathbb{B}_{\tau}(R) \subset B_{t} \quad \text { for all } \tau \leq t-t_{0}
$$


Definition 2.5 The process $\{U(t, \tau)\}_{t \geq \tau}$ is said to be pullback asymptotically compact if for any $t \in \mathbb{R}$, any bounded sequence $\left\{x_{n}\right\}_{n=1}^{\infty} \subset X_{\tau_{n}}$ and any sequence $\left\{\tau_{n}\right\}_{n=1}^{\infty}$ with $\tau_{n} \rightarrow$ $-\infty$ as $n \rightarrow \infty$, the sequence $\left\{U\left(t, \tau_{n}\right) x_{n}\right\}_{n=1}^{\infty}$ is precompact in $\left\{X_{t}\right\}_{t \in \mathbb{R}}$.

Definition 2.6 The time-dependent global attractor for the process $\{U(t, \tau)\}_{t \geq \tau}$ is the smallest family $\hat{\mathcal{A}}=\left\{\mathcal{A}_{t}\right\}_{t \in \mathbb{R}}$ such that

(i) $\mathcal{A}_{t}$ is compact in $X_{t}$;

(ii) $\hat{\mathcal{A}}$ is invariant, i.e., $U(t, \tau) \mathcal{A}_{\tau}=\mathcal{A}_{t}, \forall t \geq \tau$;

(iii) $\hat{\mathcal{A}}$ is pullback attracting, i.e., it is uniformly bounded and the limit

$$
\lim _{\tau \rightarrow-\infty} \delta_{t}\left(U(t, \tau) C_{\tau}, \mathcal{A}_{t}\right)=0
$$

holds for every uniformly bounded family $\hat{C}=\left\{C_{t}\right\}_{t \in \mathbb{R}}$ and every fixed $t \in \mathbb{R}$.

Remark 2.7 The attracting property can be equivalently stated in terms of pullback absorbing: a (uniformly bounded) family $\mathcal{K}=\left\{K_{t}\right\}_{t \in \mathbb{R}}$ is called pullback attracting if for any $\epsilon>0$ the family $\left\{\mathcal{O}_{t}^{\epsilon}\left(K_{t}\right)\right\}_{t \in \mathbb{R}}$ is pullback absorbing.

Similarly to Theorem 4.2 in [8], we have the following theorem.

Theorem 2.8 The time-dependent global attractor $\hat{\mathcal{A}}$ exists and it is unique if and only if the process $\{U(t, \tau)\}_{t \geq \tau}$ is asymptotically compact, namely, the set

$$
\mathbb{K}=\left\{\mathcal{K}=\left\{K_{t}\right\}_{t \in \mathbb{R}}: K_{t} \subset X_{t} \text { is compact }, \mathbb{K} \text { is pullback attracting }\right\}
$$

is not empty.

\subsection{Some results}

In order to obtain the time-dependent global attractors of Eq. (1.1), we need the following definitions and conclusions, which are similar to those in $[6,13,14,19,22,27]$.

Definition 2.9 Let $\left\{X_{t}\right\}_{t \in \mathbb{R}}$ be a family of Banach spaces and $\hat{C}=\left\{C_{t}\right\}_{t \in \mathbb{R}}$ be a family of uniformly bounded subset of $\left\{X_{t}\right\}_{t \in \mathbb{R}}$. We call a function $\psi_{\tau}^{t}(\cdot, \cdot)$, defined on $\left\{X_{t}\right\}_{t \in \mathbb{R}} \times\left\{X_{t}\right\}_{t \in \mathbb{R}}$, a contractive function on $C_{\tau} \times C_{\tau}$ if for fixed $t \in \mathbb{R}$ and any sequence $\left\{x_{n}\right\}_{n=1}^{\infty} \subset C_{\tau}$, there is a subsequence $\left\{x_{n_{k}}\right\}_{k=1}^{\infty} \subset\left\{x_{n}\right\}_{n=1}^{\infty}$ such that

$$
\lim _{k \rightarrow \infty} \lim _{l \rightarrow \infty} \psi_{\tau}^{t}\left(x_{n_{k}}, x_{n_{l}}\right)=0 \quad \text { for all } t \geq \tau .
$$

We denote the set of all contractive functions on $C_{\tau} \times C_{\tau}$ by $\operatorname{Contr}\left(C_{\tau}\right)$.

Theorem 2.10 Let $\{U(t, \tau)\}_{t \geq \tau}$ be a process on Banach spaces $\left\{X_{t}\right\}_{t \in \mathbb{R}}$ and have a pullback absorbing set $\hat{B}=\left\{B_{t}\right\}_{t \in \mathbb{R}}$. Moreover, assume that, for any $\epsilon>0$, there exist $\tau_{0}=\tau_{0}(\epsilon)<$ t and $\psi_{\tau_{0}}^{t}(\cdot, \cdot) \in \hat{C}\left(B_{\tau_{0}}\right)$ such that

$$
\left\|U\left(t, \tau_{0}\right) x-U\left(t, \tau_{0}\right) y\right\|_{X_{t}} \leq \epsilon+\psi_{\tau_{0}}^{t}(x, y), \quad \forall x, y \in B_{\tau_{0}},
$$

for any $t \in \mathbb{R}$. Then $\{U(t, \tau)\}_{t \geq \tau}$ is pullback asymptotically compact in $\left\{X_{t}\right\}_{t \in \mathbb{R}}$. 
Proof We need to prove that, for any $\left\{x_{n}\right\}_{n=1}^{\infty} \subset B_{\tau_{n}}$ and any $\tau_{n} \rightarrow-\infty$ as $n \rightarrow \infty$,

the sequence $\left\{U\left(t, \tau_{n}\right) x_{n}\right\}_{n=1}^{\infty}$ is precompact in $\left\{X_{t}\right\}_{t \in \mathbb{R}}$.

In the following, we will show that $\left\{U\left(t, \tau_{n}\right) x_{n}\right\}_{n=1}^{\infty}$ has a convergent subsequence via diagonal methods.

Taking $\epsilon_{m}>0$ with $\epsilon_{m} \rightarrow 0$ as $m \rightarrow \infty$.

Then, for $\epsilon_{1}>0$, by the assumptions, there exist $\tau_{0}=\tau_{0}\left(\epsilon_{1}\right)<t$ and $\psi_{\tau_{0}}^{t}(\cdot, \cdot) \in \hat{C}\left(B_{\tau_{0}}\right)$ such that

$$
\left\|U\left(t, \tau_{0}\right) x-U\left(t, \tau_{0}\right) y\right\|_{X_{t}} \leq \epsilon_{1}+\psi_{\tau_{0}}^{t}(x, y), \quad \forall x, y \in B_{\tau_{0}}
$$

for any $t \in \mathbb{R}$, where $\psi_{\tau_{0}}^{t}$ depends on $\tau_{0}$.

Since $\tau_{n} \rightarrow-\infty$, without loss of generality, we assume that $\tau_{n} \leq \tau_{0}$ such that $U\left(\tau_{0}, \tau_{n}\right) x_{n} \in$ $B_{\tau_{0}}$ for each $n \in \mathbb{N}$. Set $y_{n}=U\left(\tau_{0}, \tau_{n}\right) x_{n}$, then from (2.1) we have

$$
\begin{aligned}
\left\|U\left(t, \tau_{n}\right) x_{n}-U\left(t, \tau_{m}\right) x_{m}\right\|_{X_{t}} & =\left\|U\left(t, \tau_{0}\right) U\left(\tau_{0}, \tau_{n}\right) x_{n}-U\left(t, \tau_{0}\right) U\left(\tau_{0}, \tau_{m}\right) x_{m}\right\|_{X_{t}} \\
& =\left\|U\left(t, \tau_{0}\right) y_{n}-U\left(t, \tau_{0}\right) y_{m}\right\|_{X_{t}} \\
& \leq \epsilon_{1}+\psi_{\tau_{0}}^{t}\left(y_{n}, y_{m}\right) .
\end{aligned}
$$

By the definition of $\hat{C}\left(B_{\tau_{0}}\right)$ and $\psi_{\tau_{0}}^{t} \in \hat{C}\left(B_{\tau_{0}}\right)$, we know that $\left\{y_{n}\right\}_{n=1}^{\infty}$ have a subsequence $\left\{y_{n_{k}}^{(1)}\right\}_{k=1}^{\infty}$ such that

$$
\lim _{k \rightarrow \infty} \lim _{l \rightarrow \infty} \psi_{\tau_{0}}^{t}\left(y_{n_{k}}^{(1)}, y_{n_{l}}^{(1)}\right) \leq \epsilon_{1}
$$

Similarly to [13, 22, 27], we have

$$
\begin{aligned}
\lim _{k \rightarrow \infty} \sup _{q \in \mathbb{N}}\left\|U\left(t, \tau_{n_{k+q}}^{(1)}\right) x_{n_{k+q}}^{(1)}-U\left(t, \tau_{n_{k}}^{(1)}\right) x_{n_{k}}^{(1)}\right\|_{X_{t}} \\
\leq \lim _{k \rightarrow \infty} \sup _{q \in \mathbb{N}} \limsup _{l \rightarrow \infty}\left\|U\left(t, \tau_{n_{k+q}}^{(1)}\right) x_{n_{k+q}}^{(1)}-U\left(t, \tau_{n_{l}}^{(1)}\right) x_{n_{l}}^{(1)}\right\|_{X_{t}} \\
\quad+\limsup _{k \rightarrow \infty} \limsup _{l \rightarrow \infty}\left\|U\left(t, \tau_{n_{k}}^{(1)}\right) x_{n_{k}}^{(1)}-U\left(t, \tau_{n_{l}}^{(1)}\right) x_{n_{l}}^{(1)}\right\|_{X_{t}} \\
\leq \epsilon_{1}+\lim _{k \rightarrow \infty} \sup _{q \in \mathbb{N}} \limsup _{l \rightarrow \infty} \psi_{\tau_{0}}^{t}\left(y_{n_{k+q}}^{(1)}, y_{n_{l}}^{(1)}\right)+\epsilon_{1}+\lim _{k \rightarrow \infty} \lim _{l \rightarrow \infty} \psi_{\tau_{0}}^{t}\left(y_{n_{k}}^{(1)}, y_{n_{l}}^{(1)}\right),
\end{aligned}
$$

which, combining with (2.2) and (2.3), implies that

$$
\lim _{k \rightarrow \infty} \sup _{q \in \mathbb{N}}\left\|U\left(t, \tau_{n_{k+q}}^{(1)}\right) x_{n_{k+q}}^{(1)}-U\left(t, \tau_{n_{k}}^{(1)}\right) x_{n_{k}}^{(1)}\right\|_{X_{t}} \leq 4 \epsilon_{1} .
$$

Therefore, there exists a $K_{1} \in \mathbb{N}$ such that

$$
\lim _{k \rightarrow \infty} \sup _{q \in \mathbb{N}}\left\|U\left(t, \tau_{n_{k}}^{(1)}\right) x_{n_{k}}^{(1)}-U\left(t, \tau_{n_{l}}^{(1)}\right) x_{n_{l}}^{(1)}\right\|_{X_{t}} \leq 5 \epsilon_{1}, \quad \text { for all } k, l \geq K_{1} .
$$


By induction, we can obtain that, for each $m \geq 1$, there exists a subsequence $\{U(t$, $\left.\left.\tau_{n_{k}}^{(m+1)}\right) x_{n_{k}}^{(m+1)}\right\}_{k=1}^{\infty}$ of $\left\{U\left(t, \tau_{n_{k}}^{(m)}\right) x_{n_{k}}^{(m)}\right\}_{k=1}^{\infty}$ and certain $K_{m+1}$ such that

$$
\lim _{k \rightarrow \infty} \sup _{q \in \mathbb{N}}\left\|U\left(t, \tau_{n_{k}}^{(m+1)}\right) x_{n_{k}}^{(m+1)}-U\left(t, \tau_{n_{l}}^{(m+1)}\right) x_{n_{l}}^{(m+1)}\right\|_{X_{t}} \leq 5 \epsilon_{m+1}, \quad \text { for all } k, l \geq K_{m+1} .
$$

Now, we consider the diagonal subsequence $\left\{U\left(t, \tau_{n_{k}}^{(k)}\right) x_{n_{k}}^{(k)}\right\}_{k=1}^{\infty}$. Since for each $m \in \mathbb{N}$, $\left\{U\left(t, \tau_{n_{k}}^{(k)}\right) x_{n_{k}}^{(k)}\right\}_{k=m}^{\infty}$ is a subsequence of $\left\{U\left(t, \tau_{n_{k}}^{(k)}\right) x_{n_{k}}^{(k)}\right\}_{k=1}^{\infty}$, then

$$
\lim _{k \rightarrow \infty} \sup _{q \in \mathbb{N}}\left\|U\left(t, \tau_{n_{k}}^{(k)}\right) x_{n_{k}}^{(k)}-U\left(t, \tau_{n_{l}}^{(l)}\right) x_{n_{l}}^{(l)}\right\|_{X_{t}} \leq 6 \epsilon_{m}, \quad \text { for all } k, l \geq \max \left\{m, K_{m}\right\}
$$

which combining with $\epsilon_{m} \rightarrow 0$ as $m \rightarrow \infty$, implies that $\left\{U\left(t, \tau_{n_{k}}^{(k)}\right) x_{n_{k}}^{(k)}\right\}_{k=1}^{\infty}$ is a Cauchy sequence in $\left\{X_{t}\right\}_{t \in \mathbb{R}}$. This shows that $\left\{U\left(t, \tau_{n}\right) x_{n}\right\}_{n=1}^{\infty}$ is precompact in $\left\{X_{t}\right\}_{t \in \mathbb{R}}$.

Similarly to Theorem 3.3 in [19], we have the following conclusion, which will be used to verify the existence of the time-dependent global attractor.

Theorem 2.11 Let $\{U(t, \tau)\}_{t \geq \tau}$ be a process on Banach space $\left\{X_{t}\right\}_{t \in \mathbb{R}}$, then $\{U(t, \tau)\}_{t \geq \tau}$ has a time-dependent global attractor in $\left\{X_{t}\right\}_{t \in \mathbb{R}}$ if the following conditions hold:

(i) $\{U(t, \tau)\}_{t \geq \tau}$ has a pullback absorbing set $\hat{B}=\left\{B_{t}\right\}_{t \in \mathbb{R}}$ in $\left\{X_{t}\right\}_{t \in \mathbb{R}}$;

(ii) $\{U(t, \tau)\}_{t \geq \tau}$ is pullback asymptotically compact in $\hat{B}=\left\{B_{t}\right\}_{t \in \mathbb{R}}$.

\section{Time-dependent global attractors}

In this section, we will establish the existence of the time-dependent global attractors.

\subsection{Existence and uniqueness of solutions}

In this subsection, we consider the well-posedness of the solutions for Eq. (1.1) with (1.4)(1.5). At first, we define the weak solutions as follows.

Definition 3.1 A weak solution of Eq. (1.1) is a function $u \in C\left([\tau, T]\right.$; $\left.\mathcal{H}_{t}\right) \cap L^{2}(\tau, T$; $\left.H_{0}^{1}(\Omega)\right) \cap L^{p}\left(\tau, T ; L^{p}(\Omega)\right)$ for all $T>\tau$, with $u(\tau)=u_{\tau}$ and such that, for all $\varphi \in H_{0}^{1}(\Omega)$, it satisfies

$$
\begin{aligned}
& \frac{d}{d t}[(u(t), \varphi)+\varepsilon(t)(\nabla u(t), \nabla \varphi)]+\left(1-\varepsilon^{\prime}(t)\right)(\nabla u(t), \nabla \varphi)+(f(u(t)), \varphi) \\
& \quad=(g(x), \varphi), \quad \text { in } \mathcal{D}^{\prime}(\tau,+\infty)
\end{aligned}
$$

Remark 3.2 We notice that, if $u(t)$ is a weak solution of Eq. (1.1), then it satisfies the energy equality

$$
\begin{gathered}
\|u(t)\|_{2}^{2}+\varepsilon(t)\|\nabla u(t)\|_{2}^{2}+\int_{s}^{t}\left(2-\varepsilon^{\prime}(r)\right)\|\nabla u(r)\|_{2}^{2} d r+2 \int_{s}^{t}(f(u(r)), u(r)) d r \\
=\|u(s)\|_{2}^{2}+\varepsilon(s)\|\nabla u(s)\|_{2}^{2}+2 \int_{s}^{t}(g(r), u(r)) d r \quad \text { for all } \tau \leq s \leq t .
\end{gathered}
$$

The following theorem gives the existence of the weak solutions, which is similar to that in [10] and can be obtained by the Faedo-Galerkin methods. 
Theorem 3.3 Let $f$ satisfy (1.4)-(1.5), $g \in H^{-1}(\Omega)$ and $u_{\tau} \in \mathcal{H}_{\tau}$. Then, for any $\tau \in \mathbb{R}$ and $t>\tau$, there exists a weak solution $u(t)$ to Eq. (1.1), which satisfies $u \in C\left([\tau, t] ; \mathcal{H}_{t}\right) \cap$ $L^{2}\left(\tau, t ; H_{0}^{1}(\Omega)\right) \cap L^{p}\left(\tau, t ; L^{p}(\Omega)\right), u_{t} \in L^{2}\left(\tau, t ; \mathcal{H}_{t}\right)$.

Proof Let $\left\{w_{j}\right\}_{j \geq 1} \subset H_{0}^{1}(\Omega) \cap L^{p}(\Omega)$ be a Hilbert basis of $L^{2}(\Omega)$ such that $\operatorname{span}\left\{w_{j}\right\}_{j \geq 1}$ is dense in $H_{0}^{1}(\Omega) \cap L^{p}(\Omega)$. In order to establish the existence of the weak solutions, we need the approximate system for any $m \geq n$ seeking $\tilde{u}^{m}(t, x)=\Sigma_{j=1}^{m} \gamma_{m j}(t) \omega_{j}(x)$ that satisfies

$$
\left\{\begin{array}{l}
\frac{d}{d t}\left[\left(\tilde{u}^{m}(t), \omega_{j}\right)+\varepsilon(t)\left(\nabla \tilde{u}^{m}(t), \nabla \omega_{j}\right)\right]+\left(1-\varepsilon^{\prime}(t)\right)\left(\nabla \tilde{u}^{m}(t), \nabla \omega_{j}\right)+\left(f\left(\tilde{u}^{m}(t)\right), \omega_{j}\right) \\
\quad=\left(g(x), \omega_{j}\right), \\
\tilde{u}_{\tau}^{m}=u_{\tau},
\end{array}\right.
$$

for a.e. $t>\tau, 1 \leq j \leq m$.

We will provide a priori estimates that show that these solutions are well-defined in the interval $[\tau, t]$ for any $t>\tau$.

Step 1: First a priori estimates. Multiplying each equation in the above system by $\gamma_{m j}(t)$, respectively, and summing from $j=1$ to $m$, we obtain

$$
\begin{aligned}
& \frac{1}{2} \frac{d}{d t}\left(\left\|\tilde{u}^{m}(t)\right\|_{2}^{2}+\varepsilon(t)\left\|\nabla \tilde{u}^{m}(t)\right\|_{2}^{2}\right)+\left(1-\varepsilon^{\prime}(t)\right)\left\|\nabla \tilde{u}^{m}(t)\right\|_{2}^{2} \\
& \quad+\left(f\left(\tilde{u}^{m}(t)\right), \tilde{u}^{m}(t)\right)=\left(g(x), \tilde{u}^{m}(t)\right) \leq \frac{1}{2}\|g\|_{H^{-1}}^{2}+\frac{1}{2}\left\|\nabla \tilde{u}^{m}(t)\right\|_{2}^{2} \text {, a.e. } t>\tau,
\end{aligned}
$$

where we have used the Hölder and Young inequalities.

Furthermore, by (1.5), we know that

$$
\begin{aligned}
& \frac{d}{d t}\left(\left\|\tilde{u}^{m}(t)\right\|_{2}^{2}+\varepsilon(t)\left\|\nabla \tilde{u}^{m}(t)\right\|_{2}^{2}\right)+\left(1-2 \varepsilon^{\prime}(t)\right)\left\|\nabla \tilde{u}^{m}(t)\right\|_{2}^{2}+2 c_{1}\left\|\tilde{u}^{m}(t)\right\|_{p}^{p} \\
& \quad \leq 2 c_{0}|\Omega|+\|g\|_{H^{-1}}^{2}, \quad \text { a.e. } t>\tau .
\end{aligned}
$$

Integrating it in $[\tau, t]$, we have

$$
\begin{gathered}
\left\|\tilde{u}^{m}(t)\right\|_{2}^{2}+\varepsilon(t)\left\|\nabla \tilde{u}^{m}(t)\right\|_{2}^{2}+\int_{\tau}^{t}\left(1-2 \varepsilon^{\prime}(s)\right)\left\|\nabla \tilde{u}^{m}(s)\right\|_{2}^{2} d s+2 c_{1} \int_{\tau}^{t}\left\|\tilde{u}^{m}(s)\right\|_{p}^{p} d s \\
\leq\left\|\tilde{u}^{m}(\tau)\right\|_{2}^{2}+\varepsilon(\tau)\left\|\nabla \tilde{u}^{m}(\tau)\right\|_{2}^{2}+\left(2 c_{0}|\Omega|+\|g\|_{H^{-1}}^{2}\right)(t-\tau) \quad \text { for all } t \geq \tau .
\end{gathered}
$$

Hence,

$$
\begin{aligned}
& \left\|\tilde{u}^{m}(t)\right\|_{2}^{2}+\varepsilon(t)\left\|\nabla \tilde{u}^{m}(t)\right\|_{2}^{2}+\int_{\tau}^{t}\left\|\nabla \tilde{u}^{m}(s)\right\|_{2}^{2} d s+2 c_{1} \int_{\tau}^{t}\left\|\tilde{u}^{m}(s)\right\|_{p}^{p} d s \\
& \quad \leq\left\|\tilde{u}^{m}(\tau)\right\|_{2}^{2}+\varepsilon(\tau)\left\|\nabla \tilde{u}^{m}(\tau)\right\|_{2}^{2}+\left(2 c_{0}|\Omega|+\|g\|_{H^{-1}}^{2}\right)(t-\tau) \quad \text { for all } t \geq \tau .
\end{aligned}
$$

So, from (3.1), we can get

$$
\left\{\tilde{u}^{m}\right\}_{m \geq n} \text { is bounded in } L^{\infty}\left(\tau, t ; \mathcal{H}_{t}\right) \cap L^{2}\left(\tau, t ; H_{0}^{1}(\Omega)\right) \cap L^{p}\left(\tau, t ; L^{p}(\Omega)\right)
$$

for all $t>\tau$. 
Moreover, combining with (1.5) and (3.2), we obtain

$$
\left\{f\left(\tilde{u}^{m}\right)\right\}_{m \geq n} \text { is bounded in } L^{q}\left(\tau, t ; L^{q}(\Omega)\right) \text { for all } t>\tau,
$$

where $q=p /(p-1)$.

Then there exist functions $\tilde{u} \in L^{\infty}\left(\tau, t ; \mathcal{H}_{t}\right) \cap L^{2}\left(\tau, t ; H_{0}^{1}(\Omega)\right) \cap L^{p}\left(\tau, t ; L^{p}(\Omega)\right)$ and $\tilde{\chi} \in$ $L^{q}\left(\tau, t ; L^{q}(\Omega)\right)$ for all $t>\tau$, and a subsequence such that

$$
\begin{cases}\tilde{u}^{m} \rightarrow \tilde{u} & \text { weakly-star in } L^{\infty}\left(\tau, t ; \mathcal{H}_{t}\right), \\ \tilde{u}^{m} \rightarrow \tilde{u} & \text { weakly in } L^{2}\left(\tau, t ; H_{0}^{1}(\Omega)\right), \\ \tilde{u}^{m} \rightarrow \tilde{u} & \text { weakly in } L^{p}\left(\tau, t ; L^{p}(\Omega)\right), \\ f\left(\tilde{u}^{m}\right) \rightarrow \tilde{\chi} & \text { weakly in } L^{q}\left(\tau, t ; L^{q}(\Omega)\right) .\end{cases}
$$

Step 2: Uniform estimate for the time derivatives. Multiplying each equation of the approximate system by $\gamma_{m j}^{\prime}(t)$ and summing from $j=1$ to $m$, we arrive at

$$
\begin{aligned}
& \left\|\left(\tilde{u}^{m}\right)^{\prime}(t)\right\|_{2}^{2}+\varepsilon(t)\left\|\left(\nabla \tilde{u}^{m}\right)^{\prime}(t)\right\|_{2}^{2}+\frac{1}{2} \frac{d}{d t}\left\|\nabla \tilde{u}^{m}(t)\right\|_{2}^{2} \\
& +\left(f\left(\tilde{u}^{m}\right),\left(\tilde{u}^{m}\right)^{\prime}(t)\right)=\left(g(x),\left(\tilde{u}^{m}\right)^{\prime}(t)\right), \quad \text { a.e. } t>\tau .
\end{aligned}
$$

By the Hölder and Young inequalities, we have

$$
\begin{gathered}
\left\|\left(\tilde{u}^{m}\right)^{\prime}(t)\right\|_{2}^{2}+2 \varepsilon(t)\left\|\left(\nabla \tilde{u}^{m}\right)^{\prime}(t)\right\|_{2}^{2}+\frac{d}{d t}\left\|\nabla \tilde{u}^{m}(t)\right\|_{2}^{2} \\
+2 \frac{d}{d t} \int_{\Omega} \mathcal{F}\left(\tilde{u}^{m}(t, x)\right) d x \leq\|g\|_{2}^{2}, \quad \text { a.e. } t>\tau .
\end{gathered}
$$

Integrating it from $\tau$ to $t$, and from (1.6) we can get

$$
\begin{aligned}
& \left\|\nabla \tilde{u}^{m}(t)\right\|_{2}^{2}+2 \tilde{c}_{1}\left\|\tilde{u}^{m}(t)\right\|_{p}^{p}+\int_{\tau}^{t}\left(\left\|\left(\tilde{u}^{m}\right)^{\prime}(s)\right\|_{2}^{2}+\varepsilon(t)\left\|\left(\nabla \tilde{u}^{m}\right)^{\prime}(s)\right\|_{2}^{2}\right) d s \\
& \leq 4 \tilde{c}_{0}|\Omega|+\left\|\nabla \tilde{u}^{m}(\tau)\right\|_{2}^{2}+2 \tilde{c}_{2}\left\|\tilde{u}^{m}(\tau)\right\|_{p}^{p}+\|g\|_{2}^{2}(t-\tau)
\end{aligned}
$$

for all $t \geq \tau$ and any $m \geq n$.

Since $\tilde{u}_{\tau}^{m}=u_{\tau}$ for all $m \geq n$ and $\tilde{u}_{\tau}^{m} \in H_{0}^{1}(\Omega) \cap L^{p}(\Omega)$, by (3.4), we obtain

$$
\left\{\tilde{u}^{m}(t)\right\}_{m \geq n} \text { is bounded in } L^{\infty}\left(\tau, t ; H_{0}^{1}(\Omega) \cap L^{p}(\Omega)\right)
$$

and

$$
\left\{\left(\tilde{u}^{m}\right)^{\prime}(t)\right\}_{m \geq n} \text { is bounded in } L^{2}\left(\tau, t ; \mathcal{H}_{t}\right)
$$

for all $t>\tau$. Then there exist functions $\tilde{u} \in L^{\infty}\left(\tau, t ; H_{0}^{1}(\Omega) \cap L^{p}(\Omega)\right)$ and $\tilde{u}_{t} \in L^{2}\left(\tau, t ; \mathcal{H}_{t}\right)$ for all $t>\tau$, which improve the regularity of $\tilde{u}$ obtained in Step 1 .

For any fixed $t>\tau$, since

$$
\left\|\tilde{u}^{m}\left(t_{2}\right)-\tilde{u}^{m}\left(t_{1}\right)\right\|_{\mathcal{H}_{t}}^{2}=\left\|\tilde{u}^{m}\left(t_{2}\right)-\tilde{u}^{m}\left(t_{1}\right)\right\|_{2}^{2}+\varepsilon(t)\left\|\nabla \tilde{u}^{m}\left(t_{2}\right)-\nabla \tilde{u}^{m}\left(t_{1}\right)\right\|_{2}^{2}
$$




$$
\begin{aligned}
& =\left\|\int_{t_{1}}^{t_{2}}\left(\tilde{u}^{m}\right)^{\prime}(s) d s\right\|_{2}^{2}+\varepsilon(t)\left\|\int_{t_{1}}^{t_{2}}\left(\nabla \tilde{u}^{m}\right)^{\prime}(s) d s\right\|_{2}^{2} \\
& \leq\left(\left\|\left(\tilde{u}^{m}\right)^{\prime}\right\|_{L^{2}\left(\tau, t ; L^{2}(\Omega)\right)}^{2}+\varepsilon(t)\left\|\left(\nabla \tilde{u}^{m}\right)^{\prime}\right\|_{L^{2}\left(\tau, t ; L^{2}(\Omega)\right)}^{2}\right)\left|t_{2}-t_{1}\right| \\
& =\left\|\left(\tilde{u}^{m}\right)^{\prime}\right\|_{L^{2}\left(\tau, t ; \mathcal{H}_{t}\right)}^{2}\left|t_{2}-t_{1}\right|,
\end{aligned}
$$

for all $t_{1}, t_{2} \in[\tau, t]$, from (3.5), (3.6) and (3.7), by the Ascoli-Arzelà Theorem, and taking into account the initial data for all the sequence, we deduce that there is a subsequence such that

$$
\tilde{u}^{m} \rightarrow \tilde{u} \quad \text { in } C\left([\tau, t] ; \mathcal{H}_{t}\right)
$$

for all $t>\tau$ and a.e. in $\Omega \times(\tau, \infty)$.

Since $f \in C(\mathbb{R}, \mathbb{R})$, we conclude that $f\left(\tilde{u}^{m}\right) \rightarrow f(\tilde{u})$ a.e. in $\Omega \times(\tau, \infty)$. So, combining with (3.3) and [15] (Lemma 1.3, p. 12) we obtain $\tilde{\chi}=f(\tilde{u})$.

Thus, together with (3.3) and (3.8), by taking the limit in the equations satisfied by $\left\{\tilde{u}^{m}\right\}$ and, thanks to the fact that $\operatorname{span}\left\{\omega_{j}\right\}_{j \geq 1}$ is dense in $H_{0}^{1}(\Omega) \cap L^{p}(\Omega)$, we conclude that $\tilde{u}$ is a weak solution of Eq. (1.1).

Step 3: Proof of the general statement by density. For each $n \in \mathbb{N}$, we define $u_{\tau}^{n}=$ $\Sigma_{j=1}^{n}\left(u_{\tau}, \omega_{j}\right) \omega_{j}$. (Due to the fact that $\left\{\omega_{j}\right\}_{j \geq 1}$ is a Hilbert basis of $L^{2}(\Omega)$, it is easy to check that $u_{\tau}^{n} \rightarrow u_{\tau}$ in $\mathcal{H}_{\tau}$.)

Let also consider a sequence $\left\{g^{n}\right\}_{n=1}^{\infty} \subset L^{2}(\Omega)$ converging to $g \in H^{-1}(\Omega)$.

Denote by $u^{n}$ the corresponding solution to Eq. (1.1) with $g$ replaced by $g^{n}$ and initial data $u_{\tau}^{n}$.

Then, by the energy equality for each $u^{n}$, we have

$$
\begin{gathered}
\left\|u^{n}(t)\right\|_{2}^{2}+\varepsilon(t)\left\|\nabla u^{n}(t)\right\|_{2}^{2}+2 \int_{\tau}^{t}\left\|\nabla u^{n}(s)\right\|_{2}^{2} d s+2 \int_{\tau}^{t}\left(f\left(u^{n}(s)\right), u^{n}(s)\right) d s \\
=\left\|u^{n}(\tau)\right\|_{2}^{2}+\varepsilon(\tau)\left\|\nabla u^{n}(\tau)\right\|_{2}^{2}+2 \int_{\tau}^{t}\left(g^{n}(x), u^{n}(s)\right) d s, \quad \forall t \geq \tau .
\end{gathered}
$$

Similar to the reasoning process in Step 1, we get

$$
\left\{u^{n}\right\} \quad \text { is bounded in } L^{\infty}\left(\tau, t ; \mathcal{H}_{t}\right) \cap L^{2}\left(\tau, t ; H_{0}^{1}(\Omega)\right) \cap L^{p}\left(\tau, t ; L^{p}(\Omega)\right)
$$

for all $t>\tau$.

Now, combining with (1.5) and (3.9), we see that $\left\{f\left(u^{n}\right)\right\}$ is bounded in $L^{q}\left(\tau, t ; L^{q}(\Omega)\right)$ for all $t>\tau$.

Therefore, there exist functions $u \in L^{\infty}\left(\tau, t ; \mathcal{H}_{t}\right) \cap L^{2}\left(\tau, t ; H_{0}^{1}(\Omega)\right) \cap L^{p}\left(\tau, t ; L^{p}(\Omega)\right)$ and $\chi \in L^{q}\left(\tau, t ; L^{q}(\Omega)\right)$ for all $t>\tau$, and a subsequence such that

$$
\begin{cases}u^{n} \rightarrow u & \text { weakly-star in } L^{\infty}\left(\tau, t ; \mathcal{H}_{t}\right), \\ u^{n} \rightarrow u & \text { weakly in } L^{2}\left(\tau, t ; H_{0}^{1}(\Omega)\right), \\ u^{n} \rightarrow u & \text { weakly in } L^{p}\left(\tau, t ; L^{p}(\Omega)\right), \\ f\left(u^{n}\right) \rightarrow \chi & \text { weakly in } L^{q}\left(\tau, t ; L^{q}(\Omega)\right),\end{cases}
$$

for all $t>\tau$. 
Moreover, we may improve some of the above convergence. Taking into account the energy equality for $u^{n}-u^{m}$, we have

$$
\begin{aligned}
\left\|u^{n}(t)-u^{m}(t)\right\|_{2}^{2}+\varepsilon(t)\left\|\nabla u^{n}(t)-\nabla u^{m}(t)\right\|_{2}^{2}+\int_{\tau}^{t}\left\|\nabla u^{n}(s)-\nabla u^{m}(s)\right\|_{2}^{2} d s \\
\leq\left\|u^{n}(\tau)-u^{m}(\tau)\right\|_{2}^{2}+\varepsilon(\tau)\left\|\nabla u^{n}(\tau)-\nabla u^{m}(\tau)\right\|_{2}^{2}+2 l \int_{\tau}^{t}\left\|u^{n}(s)-u^{m}(s)\right\|_{2}^{2} d s \\
\quad+\left\|g^{n}-g^{m}\right\|_{H^{-1}}^{2}(t-\tau), \quad \forall t \geq \tau .
\end{aligned}
$$

By (3.11), we know that

$\left\{u^{n}\right\}$ is a Cauchy sequence in $C\left([\tau, t] ; \mathcal{H}_{t}\right) \cap L^{2}\left(\tau, t ; H_{0}^{1}(\Omega)\right)$ for all $t>\tau$.

Thus, we have $u^{n} \rightarrow u$ a.e. in $\Omega \times(\tau, \infty)$.

Therefore, as before, combining with (3.10) and [15] (Lemma 1.3, p. 12) we obtain $\chi=$ $f(u)$; and from (3.10) we may take the limit in the equations satisfied by $u^{n}$ and conclude that $u$ is a weak solution of Eq. (1.1).

For the solutions of Eq. (1.1), the following theorem shows the uniqueness and continuity with respect to initial data.

Theorem 3.4 Letf satisfy (1.4)-(1.5), $g \in H^{-1}(\Omega)$ and $u_{\tau} \in \mathcal{H}_{\tau}$, then the weak solution of Eq. (1.1) is unique. Moreover, for every two solutions $u^{1}(t)$ and $u^{2}(t)$ (with different initial data), the following Lipschitz continuity holds:

$$
\|\omega(t)\|_{2}^{2}+\varepsilon(t)\|\nabla \omega(t)\|_{2}^{2} \leq\left(\left\|\omega_{\tau}\right\|_{2}^{2}+\varepsilon(\tau)\left\|\nabla \omega_{\tau}\right\|_{2}^{2}\right) e^{2 l(t-\tau)}, \quad \forall t \geq \tau,
$$

where $\omega(t)=u^{1}(t)-u^{2}(t)$.

Proof Let $\omega(t)=u^{1}(t)-u^{2}(t)$, then $\omega(t)$ satisfies the following equation:

$$
\begin{cases}\omega_{t}-\varepsilon(t) \Delta \omega_{t}-\Delta \omega=f\left(u^{1}\right)-f\left(u^{2}\right) & \text { in } \Omega \times(\tau, \infty), \\ \omega(x, t)=0 & \text { on } \partial \Omega \times(\tau, \infty), \\ \omega(x, \tau)=u_{\tau}^{1}-u_{\tau}^{2}, & x \in \Omega .\end{cases}
$$

Taking the $L^{2}$-inner product between (3.12) and $\omega$, and using (1.4), we have

$$
\frac{d}{d t}\left(\|\omega\|_{2}^{2}+\varepsilon(t)\|\nabla \omega\|_{2}^{2}\right)+\left(2-\varepsilon^{\prime}(t)\right)\|\nabla \omega\|_{2}^{2} \leq 2 l\|\omega\|_{2}^{2} .
$$

Then

$$
\frac{d}{d t}\left(\|\omega\|_{2}^{2}+\varepsilon(t)\|\nabla \omega\|_{2}^{2}\right) \leq 2 l\left(\|\omega\|_{2}^{2}+\varepsilon(t)\|\nabla \omega\|_{2}^{2}\right) .
$$

By the Gronwall lemma, it yields

$$
\|\omega(t)\|_{2}^{2}+\varepsilon(t)\|\nabla \omega(t)\|_{2}^{2} \leq\left(\left\|\omega_{\tau}\right\|_{2}^{2}+\varepsilon(\tau)\left\|\nabla \omega_{\tau}\right\|_{2}^{2}\right) e^{2 l(t-\tau)},
$$

and the uniqueness holds. 
Thus, we define the solution processes $\{U(t, \tau)\}_{t \geq \tau}$ in the spaces $\mathcal{H}_{t}$ as:

$$
U(t, \tau): \mathcal{H}_{\tau} \rightarrow \mathcal{H}_{t}, \quad U(t, \tau) u_{\tau}=u(t), \quad \forall t \geq \tau
$$

Moreover, Theorem 3.4 shows that the process $\{U(t, \tau)\}_{t \geq \tau}$ is Lipschitz in $\mathcal{H}_{t}$ :

$$
\left\|U(t, \tau) u_{\tau}^{1}-U(t, \tau) u_{\tau}^{2}\right\|_{\mathcal{H}_{t}} \leq\left\|u_{\tau}^{1}-u_{\tau}^{2}\right\|_{\mathcal{H}_{\tau}} e^{2 l(t-\tau)}, \quad \forall t \geq \tau
$$

\subsection{Time-dependent global attractors}

In this subsection, we will verify the existence of the time-dependent global attractors in $\mathcal{H}_{t}$ for the process $\{U(t, \tau)\}_{t \geq \tau}$ defined by (3.13).

\subsubsection{Time-dependent absorbing sets}

In the following, we will obtain the time-dependent global absorbing sets.

Lemma 3.5 Letf satisfy (1.4)-(1.5), $g \in H^{-1}(\Omega)$ and $u_{\tau} \in \mathbb{B}_{\tau}(R) \subset \mathcal{H}_{\tau}$. Then there exists a $R_{0}>0$ such that the family $\hat{B}=\left\{B_{t}\left(R_{0}\right)\right\}_{t \in \mathbb{R}}$ is a time-dependent absorbing set for the process $\{U(t, \tau)\}_{t \geq \tau}$.

Proof Multiplying (1.1) by $u(t)$ and integrating over $x \in \Omega$, we arrive at

$$
\frac{1}{2} \frac{d}{d t}\left(\|u\|_{2}^{2}+\varepsilon(t)\|\nabla u\|_{2}^{2}\right)+\left(1-\frac{1}{2} \varepsilon^{\prime}(t)\right)\|\nabla u\|_{2}^{2}+(f(u), u)=\langle g(x), u\rangle .
$$

Thanks to (1.5) and the Hölder inequality, we have

$$
\frac{d}{d t}\left(\|u\|_{2}^{2}+\varepsilon(t)\|\nabla u\|_{2}^{2}\right)+\left(1-\varepsilon^{\prime}(t)\right)\|\nabla u\|_{2}^{2}+2 c_{1}\|u\|_{p}^{p} \leq 2 c_{0}|\Omega|+\|g\|_{H^{-1}}^{2} .
$$

Furthermore, by (1.3), we can get

$$
\frac{d}{d t}\left(\|u\|_{2}^{2}+\varepsilon(t)\|\nabla u\|_{2}^{2}\right)+\frac{1}{1+L}\left(\lambda_{1}\|u\|_{2}^{2}+\varepsilon(t)\|\nabla u\|_{2}^{2}\right) \leq 2 c_{0}|\Omega|+\|g\|_{H^{-1}}^{2} .
$$

Setting $\lambda=\min \left\{\lambda_{1}, 1\right\}$ and $\beta=\frac{\lambda}{1+L}$, we deduce that

$$
\frac{d}{d t}\left(\|u\|_{2}^{2}+\varepsilon(t)\|\nabla u\|_{2}^{2}\right)+\beta\left(\|u\|_{2}^{2}+\varepsilon(t)\|\nabla u\|_{2}^{2}\right) \leq 2 c_{0}|\Omega|+\|g\|_{H^{-1}}^{2} .
$$

Multiplying (3.14) by $e^{\beta t}$ and integrating it in $[\tau, t]$, we obtain

$$
\begin{aligned}
& \left(\|u(t)\|_{2}^{2}+\varepsilon(t)\|\nabla u(t)\|_{2}^{2}\right) e^{\beta t} \\
& \quad \leq\left(\|u(\tau)\|_{2}^{2}+\varepsilon(\tau)\|\nabla u(\tau)\|_{2}^{2}\right) e^{\beta \tau}+\left(2 c_{0}|\Omega|+\|g\|_{H^{-1}}^{2}\right) \int_{\tau}^{t} e^{\beta s} d s, \quad \forall t \geq \tau .
\end{aligned}
$$

Therefore,

$$
\left(\|u(t)\|_{2}^{2}+\varepsilon(t)\|\nabla u(t)\|_{2}^{2}\right) \leq\left(\|u(\tau)\|_{2}^{2}+\varepsilon(\tau)\|\nabla u(\tau)\|_{2}^{2}\right) e^{-\beta(t-\tau)}+\frac{1}{\beta}\left(2 c_{0}|\Omega|+\|g\|_{H^{-1}}^{2}\right)
$$




$$
\leq 1+\frac{1}{\beta}\left(2 c_{0}|\Omega|+\|g\|_{H^{-1}}^{2}\right)=R_{0}
$$

provided that $t-\tau \geq t_{0}$ with $t_{0}=\frac{1}{\beta} \ln \left(\left\|u_{\tau}\right\|_{2}^{2}+\varepsilon(\tau)\left\|\nabla u_{\tau}\right\|_{2}^{2}\right)$, from which we obtain the existence of the time-dependent absorbing set.

\subsubsection{Time-dependent global attractors}

At first, we have the following lemma, which is similar to that in [15].

Lemma 3.6 Let $f$ satisfy (1.4)-(1.5), $g \in H^{-1}(\Omega), u_{\tau} \in \mathcal{H}_{\tau}$ and $\left\{u^{n}(t)\right\}_{n=1}^{\infty}$ be a sequence of solutions for Eq. (1.1) with initial data $u_{\tau}^{n} \in \mathcal{H}_{\tau}(n=1,2, \ldots)$, then there exists a subsequence of $\left\{u^{n}(t)\right\}_{n=1}^{\infty}$ that converges strongly in $L^{2}\left(\tau, t ; L^{2}(\Omega)\right)$.

Proof By (1.5) and Theorem 3.3, we know that there exists a sequence $\left\{u^{n}(t)\right\}_{n=1}^{\infty} \subset$ $L^{2}\left(\tau, T ; H_{0}^{1}(\Omega)\right),\left\{f\left(u^{n}(t)\right)\right\}_{n=1}^{\infty} \subset L^{q}\left(\tau, T ; L^{q}(\Omega)\right)$. Then, from Eq. (1.1), we obtain $\partial_{t} u^{n}-$ $\varepsilon(t) \partial_{t} \Delta u^{n}=\Delta u^{n}-f\left(u^{n}\right)+g(x) \in L^{2}\left(\tau, T ; H^{-1}(\Omega)\right)+L^{q}\left(\tau, T ; L^{q}(\Omega)\right) \subset L^{2}\left(\tau, T ; H^{-2}(\Omega)\right)$. By the regularization theory for elliptic equations, we know that $\partial_{t} u^{n} \in L^{2}\left(\tau, T ; L^{2}(\Omega)\right)$. As in [15], there exists a subsequence of $\left\{u^{n}(t)\right\}_{n=1}^{\infty}$ (still denoted by $\left\{u^{n}(t)\right\}_{n=1}^{\infty}$ ) that converges strongly in $L^{2}\left(\tau, T ; L^{2}(\Omega)\right)$.

Then we have the following theorem, which will obtain the pullback asymptotic compactness for the process $\{U(t, \tau)\}_{t \geq \tau}$ defined by (3.13).

Theorem 3.7 Letf satisfy (1.4)-(1.5), $g \in H^{-1}(\Omega)$ and $u_{\tau} \in \mathbb{B}_{\tau}(R) \subset \mathcal{H}_{\tau}$, then $\{U(t, \tau)\}_{t \geq \tau}$ is pullback asymptotically compact in $\mathcal{H}_{t}$.

Proof Let $u^{i}(t)(i=1,2)$ be the solutions corresponding to initial data $u_{\tau}^{i} \in \mathbb{B}_{\tau}(R) \subset \mathcal{H}_{\tau}$, that is, $u^{i}(t)$ satisfies the following equation:

$$
u_{t}-\varepsilon(t) \Delta u_{t}-\Delta u+f(u)=g(x), \quad \text { in } \Omega \times(\tau, \infty),
$$

with initial data

$$
u^{i}(x, \tau)=u_{\tau}^{i}, \quad x \in \Omega .
$$

Denoting $\omega(t)=u^{1}(t)-u^{2}(t)$, then $\omega(t)$ satisfies the following equation:

$$
\omega_{t}-\varepsilon(t) \Delta \omega_{t}-\Delta \omega+f\left(u^{1}\right)-f\left(u^{2}\right)=0, \quad \text { in } \Omega \times(\tau, \infty),
$$

with initial data

$$
\omega(x, \tau)=u_{\tau}^{1}-u_{\tau}^{2}, \quad x \in \Omega .
$$

Multiplying (3.15) by $\omega(t)$ and integrating it in $\Omega$, then, by (1.4), we obtain

$$
\frac{d}{d t}\left(\|\omega\|_{2}^{2}+\varepsilon(t)\|\nabla \omega\|_{2}^{2}\right)+\left(2-\varepsilon^{\prime}(t)\right)\|\nabla \omega\|_{2}^{2} \leq 2 l\|\omega\|_{2}^{2} .
$$


By the Poincaré inequality, we have

$$
\frac{d}{d t}\left(\|\omega\|_{2}^{2}+\varepsilon(t)\|\nabla \omega\|_{2}^{2}\right)+\beta_{1}\left(\|\omega\|_{2}^{2}+\varepsilon(t)\|\nabla \omega\|_{2}^{2}\right) \leq 2 l\|\omega\|_{2}^{2}
$$

where $\beta_{1}=2 \beta, \beta$ is given by (3.14).

Thanks to the Gronwall lemma, we get

$$
\begin{aligned}
& \|\omega(t)\|_{2}^{2}+\varepsilon(t)\|\nabla \omega(t)\|_{2}^{2} \\
& \quad \leq\left(\|\omega(\tau)\|_{2}^{2}+\varepsilon(\tau)\|\nabla \omega(\tau)\|_{2}^{2}\right) e^{-\beta_{1}(t-\tau)}+2 l \int_{\tau}^{t}\|\omega(s)\|_{2}^{2} d s, \quad \forall t \geq \tau .
\end{aligned}
$$

Setting

$$
\psi_{\tau}^{t}\left(u_{\tau}^{1}, u_{\tau}^{2}\right)=2 l \int_{\tau}^{t}\|\omega(s)\|_{2}^{2} d s
$$

combining with Definition 2.9 and Lemma 3.6, we know that $\psi_{\tau}^{t}(\cdot, \cdot)$ is a contractive function. Then, for any $\epsilon>0$ and any fixed $t \in \mathbb{R}$, let $\tau_{0}=t-\frac{1}{\beta_{1}} \ln \frac{\left\|w_{\tau}\right\|_{2}^{2}+\varepsilon(\tau)\left\|\nabla w_{\tau}\right\|_{2}^{2}}{\epsilon}$, we easily see that $\{U(t, \tau)\}_{t \geq \tau}$ is pullback asymptotically compact in $\mathcal{H}_{t}$ by Theorem 2.10 .

Combining with Lemma 3.5 and Theorem 3.7, we have the main result of this paper.

Theorem 3.8 Letf satisfy (1.4)-(1.5), $g \in H^{-1}(\Omega)$ and $u_{\tau} \in \mathbb{B}_{\tau}(R) \subset \mathcal{H}_{\tau}$, then $\{U(t, \tau)\}_{t \geq \tau}$ possesses a time-dependent global attractor $\hat{\mathcal{A}}=\left\{\mathcal{A}_{t}\right\}_{t \in \mathbb{R}}$ in $\mathcal{H}_{t}$; that is, $\mathcal{A}_{t}$ is compact, $\hat{\mathcal{A}}$ is nonempty, invariant in $\mathcal{H}_{t}$ and pullback attracts every bounded subset of $\mathcal{H}_{t}$ with respect to the $\mathcal{H}_{t}$-norm.

Remark 3.9 In Theorem 3.8, we have obtained the time-dependent global attractor $\hat{\mathcal{A}}=$ $\left\{\mathcal{A}_{t}\right\}_{t \in \mathbb{R}}$ in $\mathcal{H}_{t}$. From (1.2) we know that $\varepsilon(t) \rightarrow 0$ as $t \rightarrow+\infty$, then Eq. (1.1) becomes the classical reaction-diffusion equation $u_{t}-\Delta u+f(u)=g(x)$. An interesting question is about the limitation of $\mathcal{A}_{t}$ as $t \rightarrow+\infty$, that is, how to describe $\lim _{t \rightarrow+\infty} \mathcal{A}_{t}$ ? We will consider this problem in our next work.

\section{Acknowledgements}

The authors would like to thank the referee for his/her helpful comments and suggestions.

\section{Funding}

This work was supported by the NSFC (Grants No. 11601522), the Fundamental Research Funds for the Central Universities of China (Grants No. 17CX02036A) and the Province Natural Science Foundation of Hunan (Grants No. 2018JJ2416).

Availability of data and materials

Not applicable.

Competing interests

The authors declare that they have no competing interests.

Authors' contributions

All authors contributed equally to each part of this manuscript. All authors read and approved the final manuscript.

\section{Author details}

${ }^{1}$ Hunan Province Cooperative Innovation Center for the Construction and Development of Dongting Lake Ecological Economic Zone, College of Mathematics and Physics Science, Hunan University of Arts and Science, Changde, P.R. China. ${ }^{2}$ School of Mathematics and Statistics, Changsha University of Science and Technology, Changsha, P.R. China. ${ }^{3}$ College of Science, China University of Petroleum (East China), Qingdao, P.R. China. 


\section{Publisher's Note}

Springer Nature remains neutral with regard to jurisdictional claims in published maps and institutional affiliations.

\section{Received: 23 January 2020 Accepted: 4 May 2020 Published online: 13 May 2020}

\section{References}

1. Anh, C.T., Bao, T.Q.: Dynamics of non-autonomous nonclassical diffusion equations on $\mathbb{R}^{N}$. Commun. Pure Appl. Anal. $11,1231-1252(2012)$

2. Antontsev, S., Shmarev, S.: On a class of fully nonlinear parabolic equations. Adv. Nonlinear Anal. 8, 79-100 (2019)

3. Babin, A.V., Vishik, M.I.: Attractors of Evolution Equations. North-Holland, Amsterdam (1992)

4. Cao, Y., Yin, J.X., Wang, C.P.: Cauchy problems of semilinear pseudo-parabolic equations. J. Differ. Equ. 246, 4568-4590 (2009)

5. Chen, H., Tian, S.Y.: Initial boundary value problem for a class of semilinear pseudoparabolic equations with logarithmic nonlinearity. J. Differ. Equ. 258, 4424-4442 (2015)

6. Chueshov, I., Lasiecka, I.: Long-time dynamics of von Karman semi-flows with non-linear boundary/interior damping. J. Differ. Equ. 233, 42-86 (2007)

7. Conti, M., Pata, V: Asymptotic structure of the attractor for processes on time-dependent spaces. Nonlinear Anal., Real World Appl. 19, 1-10 (2014)

8. Conti, M., Pata, V., Temam, R.: Attractors for process on time-dependent spaces: applications to wave equations. J. Differ. Equ. 255, 1254-1277 (2013)

9. Di Plinio, F., Duane, G.S., Temam, R.: Time dependent attractor for the oscillon equation. Discrete Contin. Dyn. Syst. 29, $141-167(2011)$

10. García-Luengo, J., Marín-Rubio, P.: Reaction-diffusion equations with non-autonomous force in $\mathrm{H}^{-1}$ and delays under measurability conditions on the driving delay term. J. Math. Anal. Appl. 417, 80-95 (2014)

11. Ghisi, M., Gobbino, M., Haraux, A.: A concrete realization of the slow-fast alternative for a semilinear heat equation with homogeneous Neumann boundary conditions. Adv. Nonlinear Anal. 7, 375-384 (2018)

12. Ghoul, T.E., Nguyen, V.T., Zaag, H.: Construction of type I blowup solutions for a higher order semilinear parabolic equation. Adv. Nonlinear Anal. 9, 388-412 (2020)

13. Khanmamedov, A.K.: Global attractors for von Karman equations with nonlinear interior dissipation. J. Math. Anal. Appl. 318, 92-101 (2006)

14. Kloeden, P.E., Lorenz, T.: Pullback incremental attraction. Nonauton. Dyn. Syst. 1, 53-60 (2014)

15. Lions, J.L.: Quelques Méthodes de Résolution des Problèmes aux Limites Non Linéaires. Dunod, Paris (1969)

16. Liu, Y.F.: Time-dependent global attractor for the nonclassical diffusion equations. Appl. Anal. 94, 1439-1449 (2015)

17. Łukaszewicz, G.: On pullback attractors in $L^{p}$ for nonautonomous reaction-diffusion equations. Nonlinear Anal. 73, 350-357 (2010)

18. Ma, Q.Z., Wang, X.P., Xu, L.: Existence and regularity of time-dependent global attractors for the nonclassical reaction-diffusion equations with lower forcing term. Bound. Value Probl. 2016, 10 (2016)

19. Meng, F.J., Yang, M.H., Zhong, C.K.: Attractors for wave equations with nonlinear damping on time-dependent space. Discrete Contin. Dyn. Syst., Ser. B 21, 205-225 (2016)

20. Robinson, J.C.: Infinite-Dimensional Dynamical Systems: An Introduction to Dissipative Parabolic PDEs and the Theory of Global Attractors. Cambridge University Press, Cambridge (2001)

21. Song, H.T.: Pullback attractors of non-autonomous reaction-diffusion equations in $H_{0}^{1}$. J. Differ. Equ. 249, 2357-2376 (2010)

22. Sun, C.Y., Cao, D.M., Duan, J.Q.: Non-autonomous dynamics of wave equations with nonlinear damping and critical nonlinearity. Nonlinearity 19, 2645-2665 (2006)

23. Sun, C.Y., Yang, M.H.: Dynamics of the nonclassical diffusion equations. Asymptot. Anal. 59, 51-81 (2008)

24. Temam, R.: Infinite-Dimensional Dynamical Systems in Mechanics and Physics. Springer, New York (1997)

25. Wang, S.Y., Li, D.S., Zhong, C.K.: On the dynamics of a class of nonclassical parabolic equations. J. Math. Anal. Appl. 317, 565-582 (2006)

26. Xiao, Y.L.: Attractors for a nonclassical diffusion equation. Acta Math. Appl. Sin. Engl. Ser. 18, 273-276 (2002)

27. Xie, Y.Q., Li, Q.S., Zhu, K.X.: Attractors for nonclassical diffusion equations with arbitrary polynomial growth. Nonlinear Anal., Real World Appl. 31, 23-37 (2016)

28. Xu, R.Z., Lian, W., Niu, Y.: Global well-posedness of coupled parabolic systems. Sci. China Math. 63, 321-356 (2020)

29. Xu, R.Z., Su, J.: Global existence and finite time blow-up for a class of semilinear pseudoparabolic equations. J. Funct. Anal. 264, 2732-2763 (2013)

30. Zhang, F.H., Liu, Y.F.: Pullback attractors in $H^{\prime}\left(\mathbb{R}^{N}\right)$ for non-autonomous nonclassical diffusion equations. Dyn. Syst. 29 106-118 (2014)

31. Zhong, C.K., Yang, M.H., Sun, C.Y.: The existence of global attractors for the norm-to-weak continuous semigroup and application to the nonlinear reaction-diffusion equations. J. Differ. Equ. 223, 367-399 (2006)

32. Zhu, K.X., Sun, C.Y.: Pullback attractors for nonclassical diffusion equations with delays. J. Math. Phys. 56, 092703 (2015) 